\title{
A model based evaluation of the 1996-7 pertussis epidemic in the Netherlands
}

\author{
M. VAN BOVEN ${ }^{1 *}$, H. E. DE MELKER ${ }^{1}$, J. F. P. SCHELLEKENS ${ }^{2}$ \\ AND M. KRETZSCHMAR ${ }^{1}$ \\ ${ }^{1}$ Department of Infectious Diseases Epidemiology and ${ }^{2}$ Diagnostic Laboratory for Infectious Diseases and \\ Perinatal Screening, National Institute of Public Health and the Environment, PO Box 1, 3720 BA Bilthoven, \\ The Netherlands
}

(Accepted 5 April 2001)

\section{SUMMARY}

In the Netherlands a strong epidemic outbreak of pertussis took place in 1996-7. Here we investigate the possible causes of the epidemic, using an age-structured epidemic model. Motivated by the observation that during the epidemic the number of cases in vaccinated children had increased considerably compared to the preceding period, we focus on two vaccination related changes. First, we consider the possibility that the potency of the vaccine decreased so that it confers protection for a shorter period of time in newly vaccinated children. Second, we consider the possibility that at a certain point in time the duration of protection after vaccination decreased for all individuals. This may be the case if the pathogen population changed such that the current vaccine confers less protection. A comparison of the observed and simulated age-distribution of infections indicates that the second scenario is more in line with the observed pattern of the 1996-7 epidemic. We discuss the implications of this conclusion for $B$. pertussis circulation, and for the design of vaccination programmes in the face of a polymorphic $B$. pertussis population that may adapt itself to vaccination.

\section{INTRODUCTION}

Pertussis (whooping cough) is a highly infectious disease of the upper respiratory tract. Before the introduction of vaccination pertussis was one of the main causes of child morbidity in developed countries. After the introduction of mass vaccination the incidence of pertussis decreased to very low levels in the sixties and seventies. However, in the last 15 years there has been a resurgence of pertussis in many developed countries (e.g. Canada [1], USA [2-4], UK [5]).

In the Netherlands a strong epidemic outbreak of pertussis was observed in 1996-7 [6,7]; while the general incidence of pertussis according to notification

* Author for correspondence, present address: Institute for Animal Science and Health, PO Box 65, 8200 AB Lelystad, the Netherlands. data was very low from 1988 up to 1995 , less than three cases per 100000 , it increased more than tenfold in 1996 and 1997.

To illustrate this, Figure 1 shows the age-specific incidence of reported cases of pertussis according to notifications in the Netherlands in the pre-epidemic period (1988-95), and in the 1996-7 epidemic. The distributions show a distinctive pattern. The incidence is high in predominantly unvaccinated infants under 1 year of age. It is much lower in the age-classes of 1-3 years, and it reaches a second peak at 4 years of age. The figure also shows a clear-cut difference between the pre-epidemic and epidemic period. While the total incidence according to notifications increased more than tenfold in 1996-7, the incidence in newborns ( $<1$ year) increased only about fourfold. As a result, the fraction of notified cases in newborns decreased 

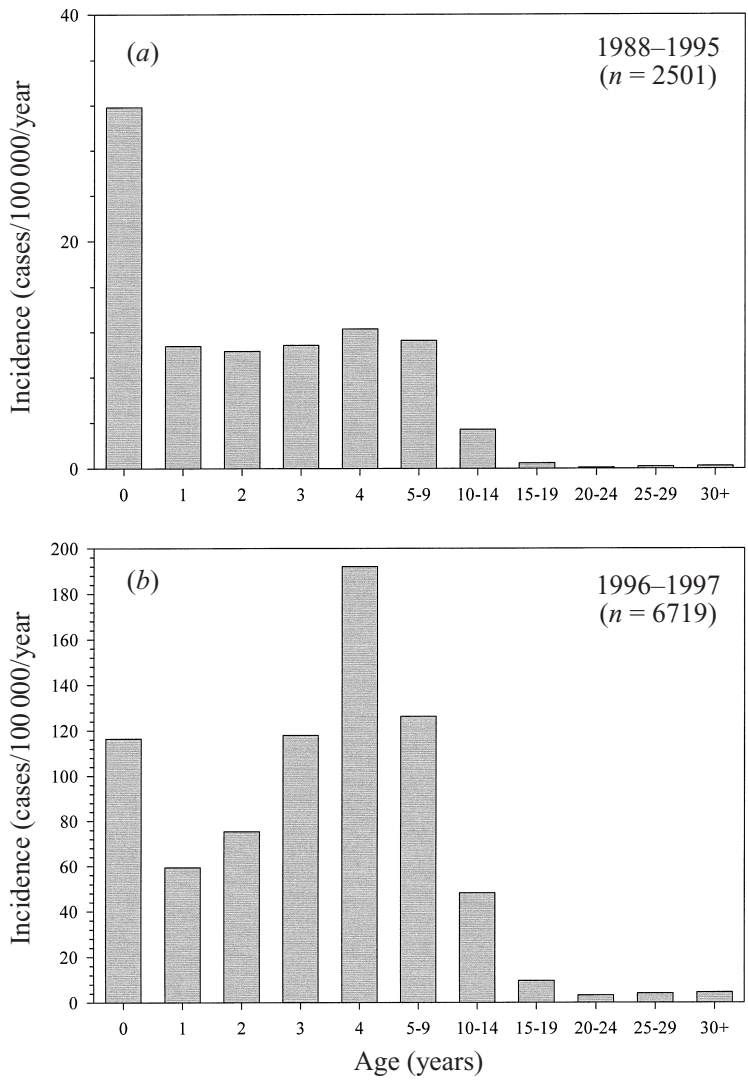

Fig. 1. Age-specific incidence of notified pertussis cases in the Netherlands. Panel (a) shows the distribution from 1988 up to 1995 (before the epidemic), while panel (b) shows the age distribution in 1996 and 1997 (during the epidemic) see [6] for details.

from $19 \%$ in the $1988-95$ period to just $6 \%$ in $1996-7$ [6].

Vaccination against pertussis is given in the first year of life. Four doses of whole-cell vaccine are administered at 3, 4, 5, and 11 months of age. Vaccination coverage in the Netherlands has been high ever since the introduction of the Dutch vaccination programme in 1953. In fact, the fraction of the population that is vaccinated has been about $96 \%$ over the years, while since 1976 the vaccination coverage has not fallen below $95 \%$ in any yearly cohort. Furthermore, the intrinsic quality of the Dutch vaccine is measured for each vaccine batch by the National Institute of Public Health and the Environment (RIVM), and meets international standards.

Nevertheless, a close analysis of the notified cases shows that a surprisingly large number of symptomatic infections took place in vaccinated infants in 1996-7 as compared to the 1988-95 period [6, 7]. In fact, in these years the incidence based on notifications was quite comparable in the vaccinated and unvaccinated populations of 4 years and older. This indicates that protection against infection after vaccination does not last lifelong [8-10].

We see two possible explanations for the sudden increase of pertussis in vaccinated children. First, it may be that the effectiveness of the vaccine decreased so that it confers immunity for a shorter period of time. In this case, one would expect that only cohorts vaccinated with the less effective vaccine are affected. Although the Dutch whole-cell vaccine meets international standards, noticeable differences between vaccine batches are sometimes observed [11-13]. Second, it has been shown that the composition of the $B$. pertussis population has changed, resulting in a possible mismatch with the currently used vaccine $[14,15]$. In that case, one would expect that all vaccinated cohorts are affected more or less equally.

In this paper we evaluate, by means of an agestructured epidemic model, the above mentioned hypotheses for the 1996-7 epidemic and the increased incidence in vaccinated infants. We present a model that makes a distinction between infection in immunologically naive individuals, and infection after vaccination or infection. Throughout we include the possibility that immunity is lost at a certain small rate.

The 1988-95 incidence data are used to estimate the so-called force of infection (the probability that a susceptible is infected in a time-period) in the preepidemic period. The method that is used is based on the underlying dynamic model, and takes into account sub-clinical infection and waning of immunity in a consistent manner [16]. Other parameters are taken from the literature, or represent educated guesses.

The analysis reveals that the second scenario, which implies a decrease in the period of immunity after vaccination for all age-classes leads to results that are more in line with the observed age-distribution of pertussis in the Netherlands in 1996-7. For this scenario already a slight increase in the rate at which immunity is lost after vaccination is sufficient to produce a sizeable epidemic if most adults were protected by vaccination in the pre-epidemic period.

\section{METHODS AND MODEL STRUCTURE}

\section{Model structure}

The main features of our model are (1) that it distinguishes between immunity conferred by vaccination or infection; and (2) that it makes a 


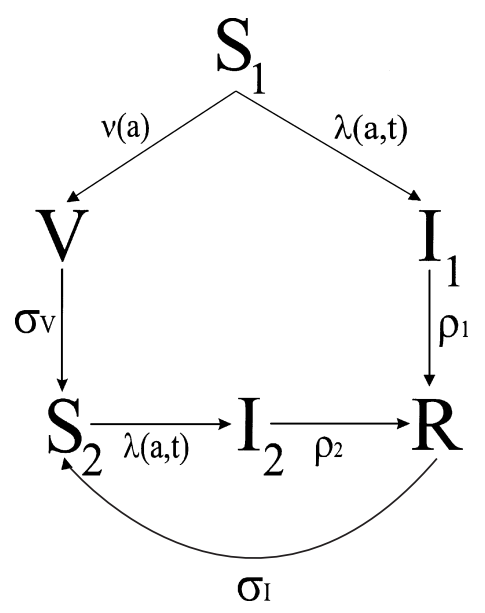

Fig. 2. Schematic structure of the model. See text for details.

distinction between infection in immunologically naive individuals (henceforth called primary infection), and infection in individuals whose immune system has been primed by infection or vaccination (secondary infection). We denote by $S_{1}$ the relative frequency of immune naive individuals. Likewise, $S_{2}$ denotes the relative frequency of susceptible individuals whose immune system has been primed before. $I_{1}$ and $I_{2}$ denote the relative frequencies of individuals with primary and secondary infection, respectively (i.e. infected from the immunologically naive or immunologically primed state). $V$ denotes the relative frequency of individuals protected against infection after vaccination, and $R$ gives the relative frequency of individuals protected after natural infection. All variables are age- and time-dependent. Figure 2 gives a schematic structure of the model.

Throughout this paper we assume that the mortality rate is zero up to the age of $L=75$ years, and that 75 years is also the maximum life span, i.e. everyone dies exactly at that age. This type of mortality is a reasonable approximation for the mortality rate in developed countries [17]. Furthermore, we assume that deaths are exactly balanced by births, so that total population size is kept constant. All newborns enter the population in the susceptible class $S_{1}$.

The rates at which a primary or secondary infection is cleared are assumed to be constant, and are denoted by $\rho_{1}$ and $\rho_{2}$, respectively. Hence, the mean infectious periods are given by $1 / \rho_{1}$ and $1 / \rho_{2}$. The constant rates at which immunity after infection or vaccination is lost are denoted by $\sigma_{I}$ and $\sigma_{V}$, respectively. In view of the absence of quantitative data, we assume that secondary infections have a fixed ratio of infectiousness relative to primary infections $\left(\beta_{2} / \beta_{1}\right)$. The age-dependent vaccination function is given by $v(a)$, and the age- and time-dependent rate at which susceptibles acquire infection (the force of infection) by $\lambda(a, t)$. The equations governing the dynamics of the model, and a brief outline on the estimation method for the force of infection are given in the Appendix.

\section{Model parameters}

For most parameters reliable estimates are virtually absent. They are therefore taken in accordance with other modelling studies [16, 19-21], or represent educated guesses. For instance, although it is known that the vaccine may not lead to effective immunity in all vaccinated individuals (i.e. primary vaccine failure), quantitative data is lacking. Here we assumed that $85 \%$ of the population is effectively vaccinated at one year of age. In view of the fact that $96 \%$ of the population is vaccinated, this means that the vaccine takes in almost $89 \%$ of the vaccinated individuals. Furthermore, although it seems plausible that primary and secondary infections may differ in some of their characteristics (e.g., infectiousness, infectious period), there is a conspicuous lack of quantitative information. We assumed that primary infections are five times more infectious than secondary infections $\left(\beta_{2} / \beta_{1}\right.$ $=0 \cdot 2$ ), and that the infectious period is about 2 weeks for both types of infection. An overview of the various parameters and their default values except those describing the contact function is given in Table 1.

Reliable estimates of the force of infection are particularly difficult to obtain. Typically, the force of infection will depend on population density, on population structure, on the number of contacts between individuals in a population, on the type of contacts, etc. It is therefore questionable whether estimates of the force of infection obtained in one population at a certain moment in time give a reasonable estimate for another population at another moment in time. Our estimates of the force of infection are obtained from the notification data of the Netherlands in the pre-epidemic period (1988-95; see Fig. 1) with the method developed in [16]. These estimates are at least consistent with the dynamic model (1). The force of infection so estimated depends on the fraction of the population vaccinated, on the duration of protection after vaccination in the preepidemic period, and on the fractions $p_{1}$ and $p_{2}$ of 
Table 1. Overview of model parameters and their default values. See Table 2 for scenarios

\begin{tabular}{|c|c|c|}
\hline Description & Parameter & Value \\
\hline $\begin{array}{l}\text { Rate of loss of immunity } \\
\text { after infection }\end{array}$ & $\sigma_{I}$ & $0 \cdot 05\left(\mathrm{yr}^{-1}\right)$ \\
\hline $\begin{array}{l}\text { Rate of loss of immunity } \\
\text { after vaccination (1998-95) }\end{array}$ & $\sigma_{V}$ & $\begin{array}{l}0 \cdot 1\left(\mathrm{yr}^{-1}\right)(\text { Scenario } 1) \\
0\left(\mathrm{yr}^{-1}\right)(\text { Scenario } 2)\end{array}$ \\
\hline $\begin{array}{l}\text { Rate of loss of immunity } \\
\text { after vaccination }(1996-\ldots)\end{array}$ & $\sigma_{V}$ & $0 \cdot 2\left(\mathrm{yr}^{-1}\right)$ (both scenarios) \\
\hline Rate of loss of primary infection & $\rho_{1}$ & $25\left(\mathrm{yr}^{-1}\right)$ \\
\hline $\begin{array}{l}\text { Rate of loss of secondary } \\
\text { infection }\end{array}$ & $\rho_{2}$ & $25\left(\mathrm{yr}^{-1}\right)$ \\
\hline $\begin{array}{l}\text { Relative infectiousness of } \\
\text { secondary infection }\end{array}$ & $\beta_{2} / \beta_{1}$ & $0 \cdot 2$ \\
\hline $\begin{array}{c}\text { Fraction of susceptibles } \\
\text { effectively vaccinated }\end{array}$ & $v$ & $0 \cdot 85$ \\
\hline Age at vaccination & $t_{v}$ & $1(\mathrm{yr})$ \\
\hline $\begin{array}{l}\text { Fraction of primary } \\
\text { infections that is notified }\end{array}$ & $p_{1}$ & $0 \cdot 1$ \\
\hline $\begin{array}{l}\text { Fraction of secondary } \\
\text { infections that is notified }\end{array}$ & $p_{2}$ & $0 \cdot 01$ \\
\hline
\end{tabular}

Table 2. Overview of the four scenarios. In Scenario 1 immunity after vaccination lasted for 10 years in the pre-epidemic period (1988-95), and in Scenario 2 it lasted lifelong in the pre-epidemic period. In Scenario A immunity after vaccination is reduced (to 5 years) for cohorts vaccinated after 1995 only, and in Scenario B immunity after vaccination is reduced for all cohorts

\begin{tabular}{|c|c|c|}
\hline & Scenario A & Scenario B \\
\hline Scenario 1 & $\begin{array}{l}\text { 1998-1995: } \sigma_{V}=0 \cdot 1 \\
1996-2020: \sigma_{V}=0 \cdot 2 \\
\text { for new cohorts }\end{array}$ & $\begin{array}{l}\text { 1988-1995: } \sigma_{V}=0 \cdot 1 \\
1996-2020: \sigma_{V}=0 \cdot 2 \\
\text { for all cohorts }\end{array}$ \\
\hline Scenario 2 & $\begin{array}{l}\text { 1988-1995: } \sigma_{V}=0 \\
1996-2020: \sigma_{V}=0 \cdot 2 \\
\quad \text { for new cohorts }\end{array}$ & $\begin{array}{l}\text { 1988-1995: } \sigma_{V}=0 \\
1996-2020: \sigma_{V}=0 \cdot 2 \\
\quad \text { for all cohorts }\end{array}$ \\
\hline
\end{tabular}

primary and secondary infections that is notified. As in our previous study [16] we assume that 1 out of 10 primary infections is notified $\left(p_{1}=0 \cdot 1\right)$, and only 1 out of 100 secondary infections $\left(p_{2}=0 \cdot 01\right)$. Although these figures are not based on any comprehensive research, they are not implausible for the situation in the Netherlands, and they are compatible with other studies [8, 9, 22-26].

Throughout this paper we will consider two scenarios (labelled 1 and 2) with respect to the preepidemic situation (1988-95), and two scenarios with respect to the type of vaccination related change in the population (labelled A and B). This gives four possible combinations, 1A, 1B, 2A, and 2B. An overview of the scenarios is given in Table 2 .

In Scenario 1 we assume that immunity after vaccination in the 1988-95 period lasted for 10 years on average $\left(\sigma_{V}=0 \cdot 1\right)$, while in Scenario 2 we assume that immunity after vaccination lasted lifelong in the pre-epidemic period $\left(\sigma_{V}=0\right)$. In view of the fact that clinically recognized pertussis cases in vaccinated individuals were rare but not absent in the preepidemic period [6-7] the second scenario probably overestimates the duration of protection by the vaccine. Although the second scenario is not the most realistic, it serves a useful purpose since it provides a baseline against which the results of the first scenario can be set. Estimates of the force of infection in the pre-epidemic period (and hence on the contact function and probabilities of infection) are given below (Fig. 3; see [16] for methods).

With respect to the type of vaccination related change, the scenarios A and B differ in the duration of protection in cohorts vaccinated before 1996. In Scenario A we assume that only for newly vaccinated cohorts the duration of protection decreases to 5 years on average $\left(\sigma_{V}=0 \cdot 2\right)$, while in Scenario B we assume that all the duration of protection decreases to 5 years for all vaccinated individuals. Scenario A applies to the situation where the effectiveness of the vaccine decreased, while Scenario B mimics the situation where the pathogen population changed, resulting in a mismatch with the vaccine. 


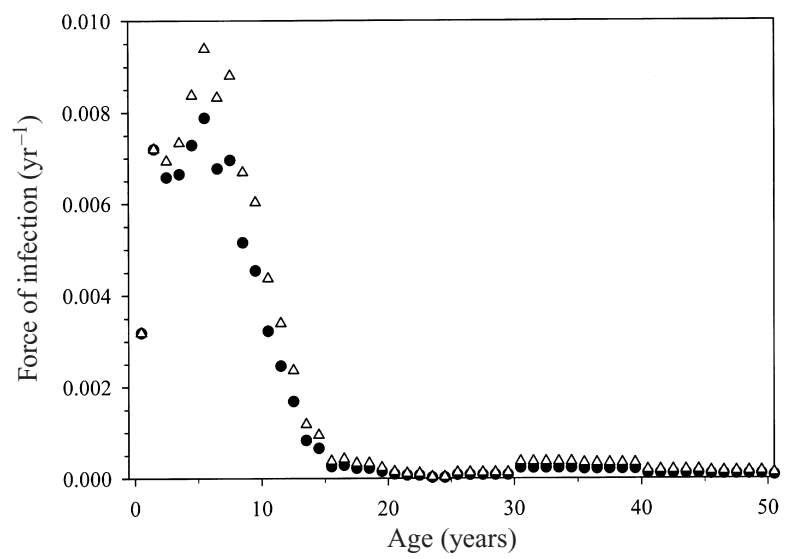

Fig. 3. Age-dependent force of infection as estimated from the 1988-95 pertussis incidence data (see Fig. 1). The solid circles show the force of infection if immunity after vaccination lasted for 10 years in the pre-epidemic period (Scenario 1), while open triangles show the force of infection if immunity is assumed to have lasted lifelong in the preepidemic period (Scenario 2).

\section{RESULTS}

\section{The pre-epidemic period (1988-95)}

Figure 3 gives the estimates of the force of infection based on the notification data of the 1988-95 period (Fig. 1). The solid circles show the results in case that immunity after vaccination lasted 10 years in the preepidemic period (Scenario 1), while the triangles show the results in case that immunity lasted lifelong in the pre-epidemic period (Scenario 2).

In both scenarios the force of infection is relatively low in newborns $\left(\approx 0.0032\left(\mathrm{yr}^{-1}\right)\right)$. It then increases to reach a peak in 5-year-old children of 0.008 in Scenario 1 and of 0.0095 in Scenario 2. In the older age-classes the force of infection decreases to low values $\left(<2 \cdot 5^{*} 10^{-4}\right.$ in Scenario 1 , and $<4 \cdot 5^{*} 10^{-4}$ in Scenario 2 ). This means that the estimated probability that a susceptible newborn is infected in a year is about $0.32 \%\left(\approx 1-e^{-0.0032}\right)$, that the probability that a susceptible 5 year old is infected in a year is $0.8 \%$ in Scenario 1 and $0.95 \%$ in Scenario 2, and that the probability of adult infection is less than $0.025 \%$ in Scenario 1 and less than $0.045 \%$ in Scenario 2 .

Figure 4 shows the age-distribution of infecteds and susceptibles 43 years after the introduction of mass vaccination. Hence, the figure corresponds to the situation in the Netherlands in 1995. Panels A (Scenario 1) and C (Scenario 2) give the age-dependent prevalence of the susceptible classes $S_{1}$ and $S_{2}$, and the infectious classes $I_{1}$ and $I_{2}$, while panels $\mathrm{B}$ and $\mathrm{D}$ give the corresponding incidence of infection. The preva- lence of primary infection in newborns (which may lead to severe complications) is relatively high, about 10 per 100000 individuals for both scenarios. Hence, about 1 out of 10000 newborns will be infectious at any given moment in time. The prevalence of primary infection decreases to relatively low values ( 2 per $100000)$ just after vaccination, and it reaches a second peak at 4 years of age $(\approx 4$ per 100000 in $\mathrm{A} ; \approx 6$ per 100000 in C). Note furthermore that in both scenarios primary infections are extremely rare in individuals older than 20 years of age.

Secondary infections typically do not lead to serious disease. They may, however, play an important role in keeping up circulation of B. pertussis. In Scenario 1 the majority of adults $(>70 \%)$ are susceptible $\left(S_{2}\right)$ in 1995. As a result, the prevalence of secondary infection might be within the range of measurement $(>0.1$ per 100000 , say) even though the force of infection on adults is rather low. In Scenario 2, on the other hand, the majority of adults $(>80 \%)$ is protected by vaccination or by previous infection. As a result, the prevalence of secondary infection is extremely low. More specifically, in Scenario 1 the prevalence of secondary infection reaches a peak of about 10 per 100000 in $6 \frac{1}{2}$ year old children, and is about 0.5 per 100000 in adults. In Scenario 2 the prevalence of secondary infection is only non-negligible $(\approx 0.5 \mathrm{per}$ 100000 ) in unvaccinated adults over 43 years of age who were born before the implementation of the Dutch mass vaccination programme in 1953.

The age-specific incidences (in cases/100000/week) as calculated from Figures $4 \mathrm{~A}$ and $4 \mathrm{C}$ are given in Figures $4 \mathrm{~B}$ and $4 \mathrm{D}$. Incidence is calculated as prevalence $\left(I_{i}\right.$ (numbers per 100000)) divided by the infectious period $\left(1 / \rho_{i}\right): \frac{1}{52} \rho_{i} I_{i}^{*}\left(100000^{-1}\right.$ wee $\left.^{-1}\right)$. Note that the figure gives the true incidence of infection rather than the observed incidence through notification. Since we assumed that 1 out of 10 primary infections is notified, and only 1 out of 100 secondary infections, the observed incidence is readily obtained by multiplying the true incidence of primary infection with $\frac{1}{10}$, the true incidence of secondary infection with $\frac{1}{100}$, and adding up the resulting numbers. In Scenario 1 the incidence of primary infection is only higher than the incidence of secondary infection in the age-classes 0-2, From age-class 3 onwards the true incidence of primary infection is higher than the true incidence of secondary infection. Hence, in these age-classes the majority $(>95 \%)$ of infections is sub-clinical and remains unnoticed. In Scenario 2, on the other hand, secondary infections are almost absent. 

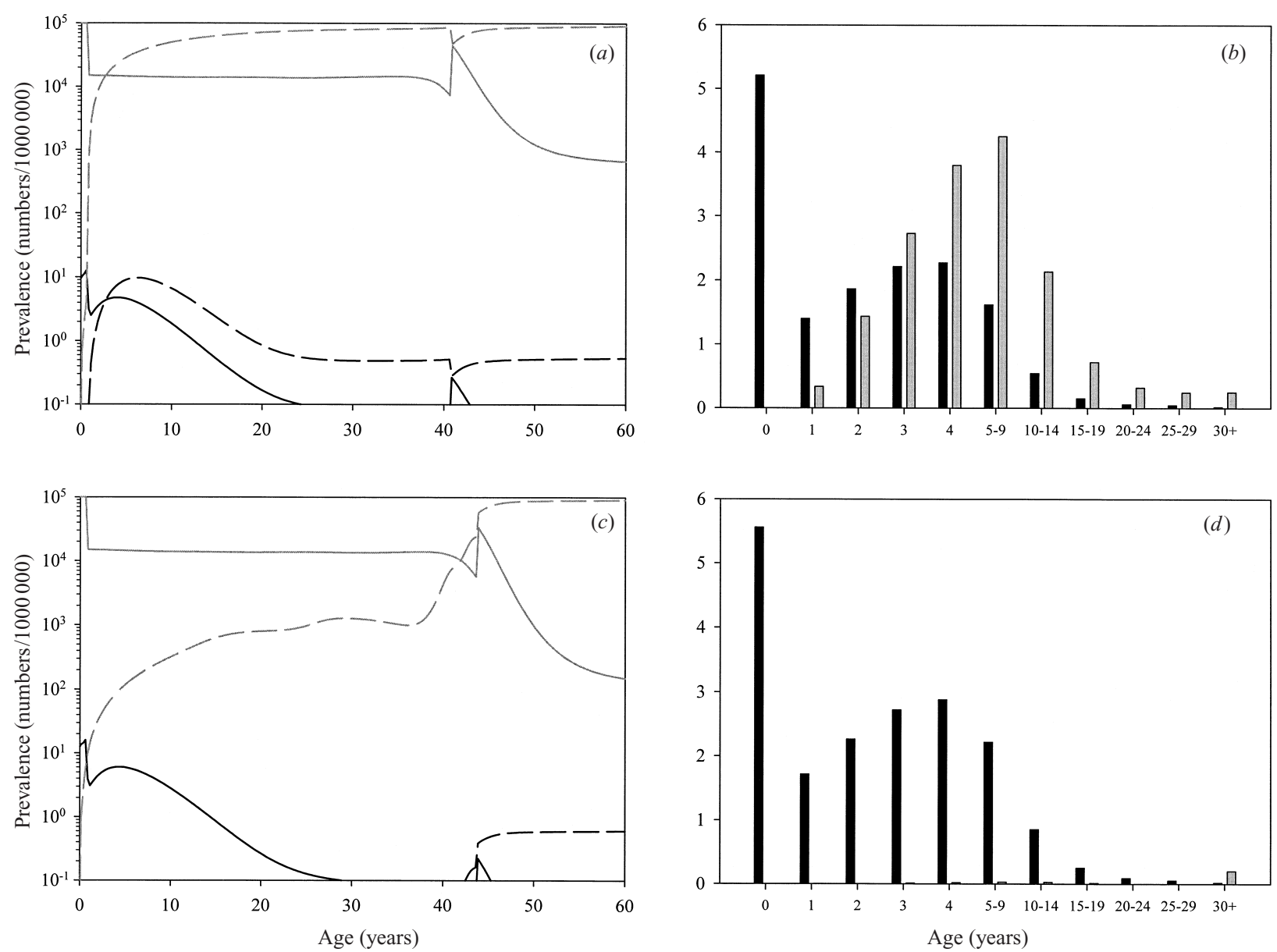

Fig. 4. The simulated situation of 1995,43 years after the introduction of the Dutch vaccination programme. Panels $(a)$ (Scenario 1) and (c) (Scenario 2) show the simulated prevalences (in numbers per 100000) by age in case that immunity after vaccination is assumed to last for 10 years and lifelong, respectively. Black lines denote the prevalence of infecteds (solid line: $I_{1}$; dashed line: $I_{2}$ ), and grey lines denote the prevalence of susceptibles (solid line: $S_{1}$; dashed line: $S_{2}$ ). Note the logarithmic scale on the ordinate. Panels $(b)$ and $(d)$ show the corresponding incidence (in cases per 100000 per week) of primary infection (black bars) and secondary infection (grey bars) by age.

With an interpretation of the pre-epidemic period at hand, we proceed to investigate the consequences of the two vaccination related changes mentioned above.

\section{Scenario A: A decrease in vaccine effectiveness}

First we investigate the consequences of a sudden decrease in the period of protection conferred by the vaccine in newly vaccinated infants only (Scenarios $1 \mathrm{~A}$ and 2A). Table 3 and Figure 5 show the results. Panels A and B of Figure 5 show time-plots of the prevalence of primary and secondary infection for the Scenarios 1A and 2A. The situation at $t=0$ in Panels A and B corresponds, roughly, to 1995. Panels C and $\mathrm{D}$ of Figure 5 show the age-specific incidence corresponding to panels $\mathrm{A}$ and $\mathrm{B}$ at the height of the first epidemic peak.
Table 3. The contribution of infections in infants of age zero as a fraction of the total number of infections in the endemic situation $\left(f_{\text {endemic }}\right)$, and at the first epidemic peak $\left(f_{\text {epidemic }}\right)$. See Table 2 for overview of the scenarios, and the main text for explanation

\begin{tabular}{ll}
\hline \hline Pre-epidemic period & First epidemic outbreak \\
\hline Scenario 1: & Scenario 1A: $f_{\text {epidemic }}=0 \cdot 17$ \\
$f_{\text {endemic }}=0 \cdot 17$ & Scenario 1B: $f_{\text {epidemic }}=0 \cdot 15$ \\
Scenario 2: & Scenario 2A: $f_{\text {epidemic }}=0 \cdot 23$ \\
$f_{\text {endemic }}=0 \cdot 29$ & Scenario 2B: $f_{\text {epidemic }}=0 \cdot 18$ \\
\hline \hline
\end{tabular}

As Figure 5 shows, a decrease in the duration of protection provided by the vaccine leads to epidemic outbreaks for both scenarios. The incidence of 

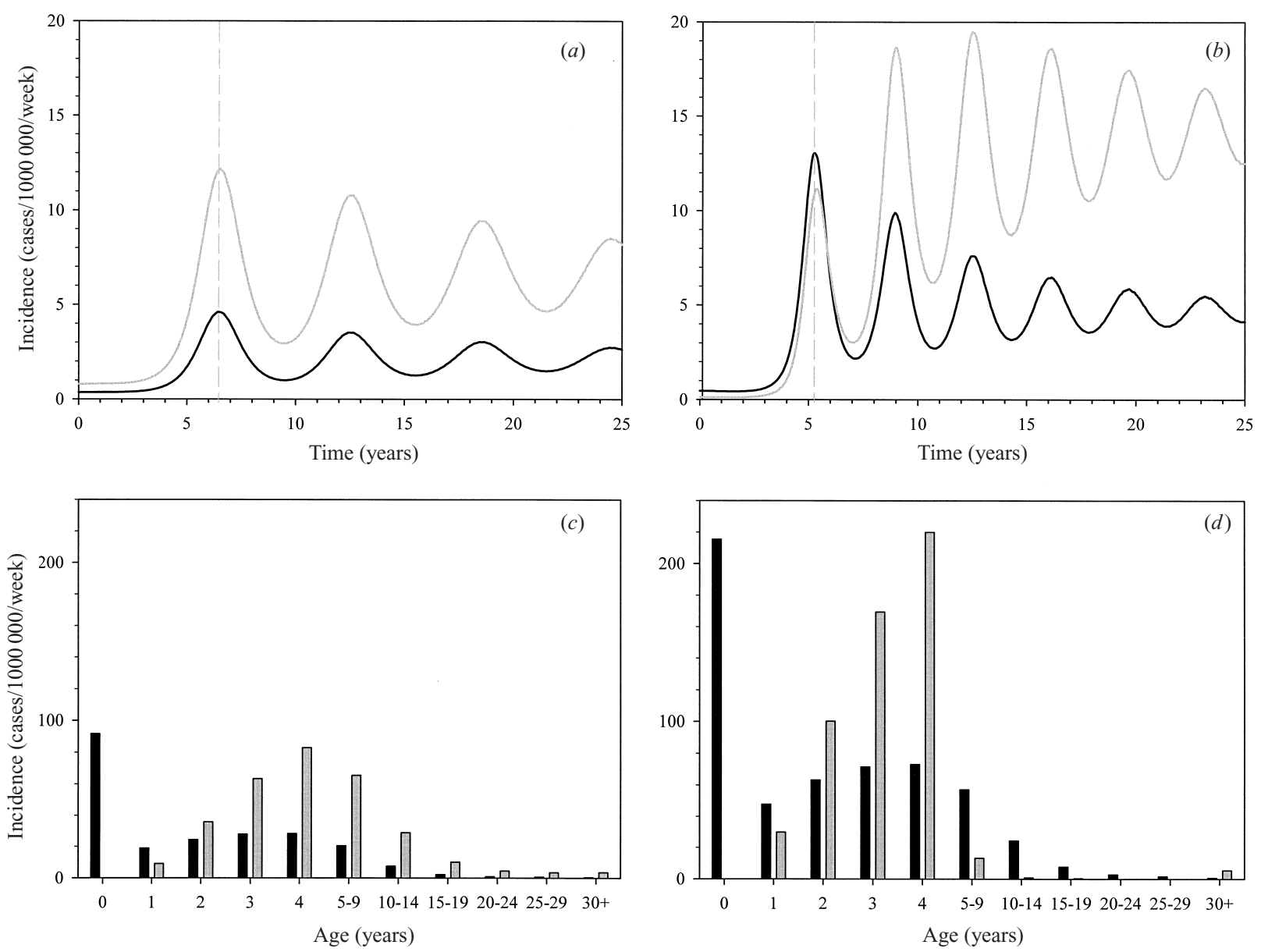

Fig. 5. Time plot of the total incidence as a function of time, and the age-specific incidence at the first epidemic peak after a reduction in the period of immunity in newly vaccinated cohorts. Panels $(a)$ and $(c)$ shows the results for Scenario $1 \mathrm{~A}$ (immunity lasted for 10 years in the endemic period), while panels $(b)$ and $(d)$ show the results for Scenario 2A (immunity lasted lifelong in the endemic period). Panels $(c)$ and $(d)$ give the simulated age-dependent incidence at the first epidemic peak $\left(t_{\text {epidemic }}\right.$ $=6.45(\mathrm{yr}$.$) in A; t_{\text {epidemic }}=5.30(\mathrm{yr}$.) in $(b))$. Note the difference in scale on the ordinate of panels $(c)$ and $(d)$ in comparison with Fig. 4.

primary and secondary infection $\left(\frac{1}{52} \rho_{i} I_{i}^{*}\left(100000^{-1}\right.\right.$ wee $\left.^{-1}\right)$ ), which in the pre-epidemic period had been $0 \cdot 36$ cases per 100000 per week, and 0.82 $\left(100000^{-1}\right.$ week $\left.^{-1}\right)$ for Scenario 1 and 0.46 and 0.13 for Scenario 2, increases to $4 \cdot 6$ and $12 \cdot 0$ at the height of the first epidemic for Scenario 1, and even to 13.0 and $11 \cdot 1$ for Scenario 2.

The figure also shows that the first epidemic outbreak occurs more than 5 years after the decrease in vaccine effectiveness has taken place (after 6.45 years in A and 5.30 years in B). This is due to the fact that the buildup of susceptibles is rather gradual. Only if enough susceptible individuals in cohorts vaccinated with the less effective vaccine have moved into those age-classes that are at the highest risk of becoming infected (1-7 years) is it possible that an epidemic may occur.

Table 3 shows how the age-distribution of cases at the height of the first epidemic peak is affected by a decrease in the period of immunity conferred by the vaccine. In particular, the table shows the contribution of infections in newborns (infants of age zero) as fraction $(f)$ of all infections before and during the epidemic. For Scenario 1 the relative contribution of infections in infants of age zero does not change at all $\left(f_{\text {endemic }}=0 \cdot 17\right.$ versus $\left.f_{\text {epidemic }}=0 \cdot 17\right)$, while for Scenario 2 the contribution of infection in newborns does decrease to some extent $\left(f_{\text {endemic }}=0.29\right.$ versus $f_{\text {epidemic }}$ $=0 \cdot 23$ ). A discussion of this phenomenon is given below.

\section{Scenario B: A change in the pathogen population}

Second, we considered a sudden decrease in the period of immunity after vaccination for all cohorts 

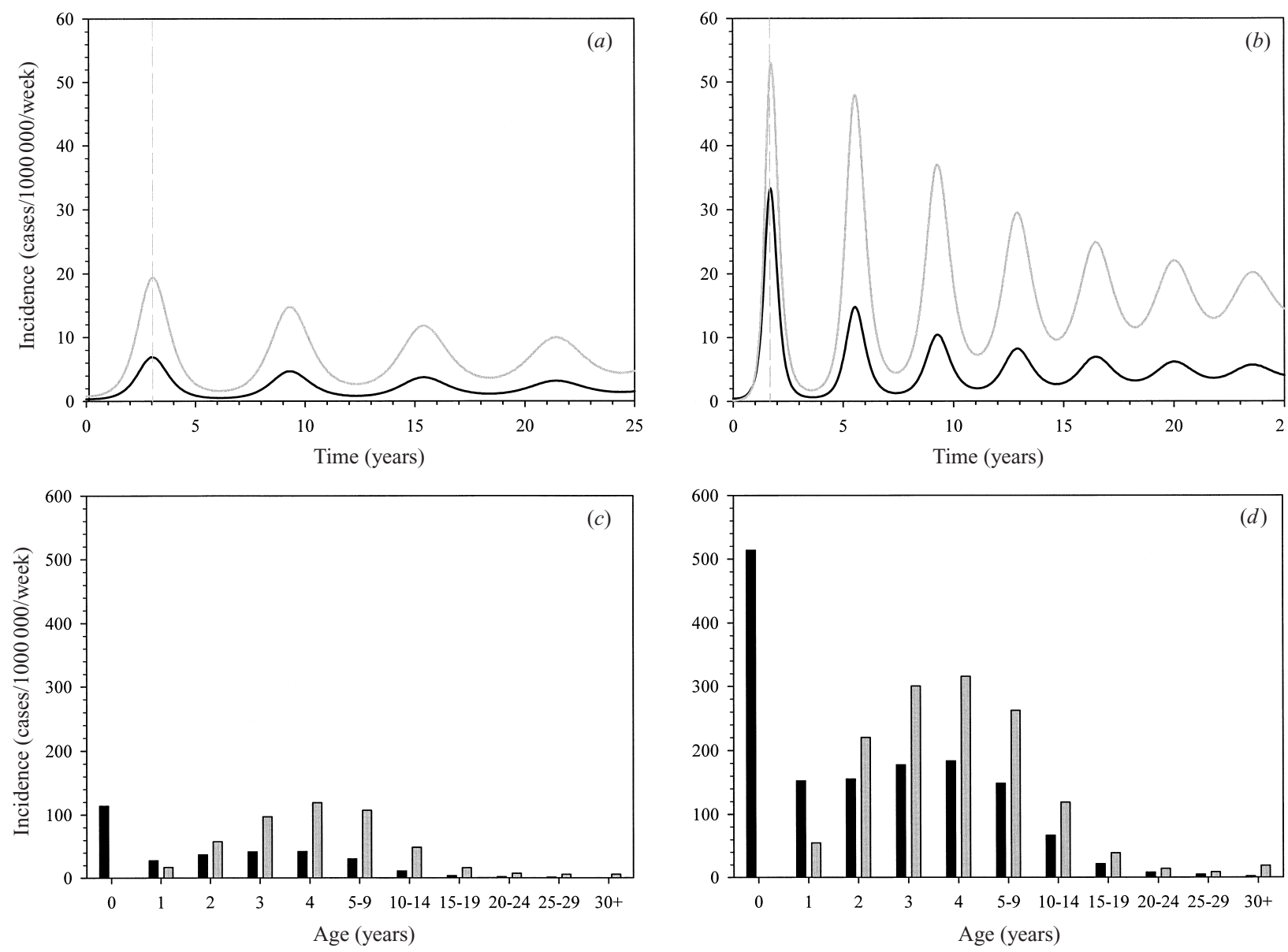

Fig. 6. Time plot of the total incidence as a function of time, and the age-specific incidence at the first epidemic peak after a reduction in the duration of immunity for all vaccinated individuals (see Fig. 5). Panels $(a)$ and $(c)$ show the results for Scenario 1B (immunity lasted for 10 years in the endemic period), while panels $(b)$ and $(d)$ show the results for Scenario $2 \mathrm{~B}$ (immunity lasted lifelong in the endemic period). Panels $(c)$ and $(d)$ give the simulated age-dependent incidence at the first epidemic peak $\left(t_{\text {epidemic }}=3.05(\mathrm{yr}\right.$.$) in (a) ; t_{\text {epidemic }}=1.70(\mathrm{yr}$.$\left.) in (b)\right)$. Note the difference in scale on the ordinates in comparison with Figures 4 and 5.

(Scenarios $1 \mathrm{~B}$ and $2 \mathrm{~B}$ ). As before, the duration of protection by vaccination is decreased from 10 years in Scenario $1\left(\sigma_{V}=0 \cdot 1\left(\mathrm{yr}^{-1}\right)\right)$ and lifelong in Scenario $2\left(\sigma_{V}=0\right)$ to 5 years $\left(\sigma_{V}=0 \cdot 2\right)$. Table 3 and Figure 6 show the results.

Figure 6 shows that a change in the pathogen population may in just a few years lead to strong recurrent epidemics. This is especially so for Scenario 2 where we assumed that the vaccine conferred lifelong immunity before the change in the pathogen population. The incidence of primary and secondary infection, which in the pre-epidemic period had been 0.36 and 0.82 cases per 100000 per week for Scenario 1 , and 0.46 and 0.13 for Scenario 2, increases to 6.7 and $19 \cdot 2$ at the height of the first epidemic for Scenario 1, and even to $33 \cdot 2$ and 52.9 for Scenario 2. In the long run $(\approx 50$ years) the population reaches a new endemic equilibrium that is at a considerably higher level than in the 1988-95 period.

The age-distribution of infections shows another striking difference between the two scenarios (Table 3). While for Scenario 1 the fraction of infections in newborns only slightly decreases $\left(f_{\text {endemic }}=0.17\right.$ to $f_{\text {epidemic }}=0 \cdot 16$, for Scenario 2 the relative contribution of transmission to newborns decreases considerably at the height of the first epidemic peak when compared to the preceding endemic period $\left(f_{\text {endemic }}=\right.$ 0.29 to $f_{\text {epidemic }}=0 \cdot 18$ ). This can be interpreted as follows. For Scenario 1 the majority of adults was already susceptible in the pre-epidemic period since immunity after vaccination lasted for only 10 years on average (Fig. 4). Hence, a decrease in the period of protection provided by the vaccine will not increase the number of susceptible adults greatly, and therefore 
cannot increase the number of infections in adults considerably. This, however, is not so for Scenario 2 where in the pre-epidemic period most adults were protected by vaccination. In fact, $85 \%$ of the population is effectively vaccinated and will be protected against infection for life. Hence, for this scenario a decrease in the period of immunity after vaccination does increase the prevalence of susceptibles considerably and, as a consequence, also increases the number of infections in adults.

\section{DISCUSSION}

\section{A comparison of scenarios}

The main aim of this paper was to compare two vaccination related changes that may have accounted for the pertussis epidemic in the Netherlands in 1996-7, and the strong increase in the number of cases in vaccinated children [6,7]. Our model results indicate that a decrease in the duration of protection by vaccination for all age-classes (corresponding to a change in the pathogen population) will always lead to a more pronounced epidemic than a decrease in the duration of protection in newly vaccinated infants only (corresponding to a change in the vaccine). This is due to the fact that a change in the pathogen population makes a potentially large reservoir of adults previously protected by vaccination available for infection in a short time-span ( $<5$ years, say). In case of a change in the vaccine the pool of susceptibles increases much slower because it is formed by newborn cohorts that have been vaccinated with a less effective vaccine. Also, it takes somewhat longer before the threshold density of susceptibles is reached that enables an epidemic to occur (5-7 years; Fig. 5).

Furthermore, a comparison of the age-distributions at the height of the first epidemic peak indicates that a shift in the age-distribution of infections towards the older age-classes is most likely to be observed if the period of immunity provided by the vaccine is reduced for all age-classes, and if the vaccine provided longlived protection in the pre-epidemic period. Again, the reason is that a large reservoir of adults previously protected by vaccination may become available for infection in a relatively short time-span, therefore shifting the balance towards the group of vaccinated individuals.

The differences between the two vaccination related changes considered here is more pronounced if a larger fraction of the population was protected by vaccination in the pre-epidemic period. Hence, the differences between the two vaccination related changes is larger if the rate at which immunity was lost in the pre-epidemic period was low, and if the fraction that was effectively vaccinated was high. If the vaccine was already imperfect in the pre-epidemic period in that it conferred immunity for a relatively short period of time (e.g. 10 years; Scenario 1), the difference between the two scenarios in the age-distribution of new infections will be rather small, and the epidemic outbreaks will be modest. If the vaccine conferred lifelong immunity in the pre-epidemic period (Scenario 2), the differences between the age-distributions age more pronounced, and the epidemic outbreaks are much stronger.

\section{Pertussis in the Netherlands}

In the Netherlands the incidence of pertussis based on notifications was low (less than 3 cases per year per $100000)$ in the pre-epidemic period (1988-95). It then suddenly increased to more than 22 cases per year per 100000 in 1996 and 1997. In the pre-epidemic period the incidence in vaccinated individuals was more than an order of magnitude lower than the incidence in unvaccinated individuals [7]. This indicates that the Dutch Whole Cell Vaccine conferred protection against severe disease for a prolonged period of time in the pre-epidemic period. In 1996-7, on the other hand, the incidence in the vaccinated and unvaccinated population of five years and older was quite comparable. This indicates that the effectiveness of the Dutch whole cell vaccine somehow decreased. This is reflected by the unusually low estimates of vaccine efficacy (based on notification data) as estimated with the screening method in 1996-7 $(<0 \cdot 3$; see $[6,7]$ for details, and $[27,28]$ for interpretation of vaccine efficacy).

A close analysis of the age-distribution of cases in the pre-epidemic and epidemic eras shows that the incidence of pertussis based on notifications in children of 3-9 years old was relatively low, about 10 per 100000 up to 1994, after which it increased to well over 100 per 100000 in 1996 and 1997, a more than tenfold increase [6,7]. Equally strong relative increases in incidence were observed in adolescents and adults. In infants of age zero, on the other hand, the relative increase in incidence in 1996-7 as compared to $1988-95$ was much less pronounced. In this ageclass the incidence increased from 32 cases per 100000 per year in $1988-95$ to 117 cases per 100000 per year 
in 1995-6, a fourfold increase. As a result, the fraction of all notified infections among infants less than 1 year old decreased from $19 \%$ in $1988-95$ to just $6 \%$ in 1996-7.

\section{Model predictions versus observed pertussis incidence}

Our model results indicate that a pronounced increase of the incidence in older vaccinated children (more than 5 years old, say) is very unlikely to be due to an increased circulation in younger vaccinated children less than 5 years old only. In fact, in our model we only observed a noticeable decrease in the fraction of infections in newborns if (1) the vast majority of individuals were protected by vaccination in the preepidemic period and if (2) the duration of the period of protection after vaccination decreased for all individuals. We may conclude that, at least qualitatively, the 1996-7 pertussis epidemic in the Netherlands is most easily explained if the vaccine had high efficacy in the pre-epidemic period (so that small changes in vaccine efficacy already lead to sizeable epidemics), and if there had been a change in the pathogen rather than a change in the vaccine (so that a noticeable shift in the age-distribution of cases towards older children will be observed). Additional support for this view comes from the fact that the shift in the agedistribution of cases is mainly due to a strong increase in the number of cases in vaccinated individuals, rather than an increased circulation in unvaccinated individuals of 1 year and older [6,7].

Quantitatively, there are some distinct differences between our model results and the 1996-7 pertussis epidemic. First, the fraction of notified infections in newborns decreased from $19 \%$ in the pre-epidemic period to just $6 \%$ during the epidemic. In our model the fraction of infections in newborns was just $17 \%$ for Scenario 1, and almost $29 \%$ for Scenario 2. This discrepancy with the observed fraction of infections in newborns may be due to our procedure to estimate the force of infection in the pre-epidemic period, to our assumptions on the contact function (i.e. proportionate mixing), and to uncertainties in model parameters (e.g. infectiousness of primary versus secondary infections, infectious period). However, varying the ratio of the infectiousness of secondary versus primary infections from $0 \cdot 1$ to $0 \cdot 5$, and the infectious period from 1 to 3 weeks did not significantly affect the results (M. van Boven, unpublished results). Most importantly, we did not observe a decrease in the fraction of infections newborns as pronounced as in the data in any of the four model scenarios considered here. This could be taken as prima facie evidence that the decrease in the protected period after vaccination may have been even stronger than considered here (from lifelong to 5 years). This, however, is at odds with the observation of waning of vaccine induced immunity before 1995, and the continuous circulation of B. pertussis before 1995 [6].

A variety of other factors may just as well account for the discrepancy between empirical data and model results. First and foremost, we assumed that the change in the composition of the B. pertussis population occurred very suddenly, at the end of 1995 . This is not very realistic. If the changes in the pathogen population occur more gradually, the difference between the scenarios are likely to become less pronounced. Clearly, the present model is not suited to answer the question how the effectiveness of the vaccine changes over time, as arbitrary choices must be made on the composition of the pathogen population. To address this question, age-structured models that explicitly include strain structure will have to be considered.

Second, there may have been an increased awareness of pertussis by the public and physicians. This may have led to an increased probability that secondary infections are recognized and notified at present [c.f. 6, 7]. Furthermore, we assumed that the degree of infectiousness, the probability of an infection remaining sub-clinical, and the probability of notification are not directly related to age. This is not very plausible. Although it is generally assumed that infections in newborns are more likely to be notified than infections in adolescents and adults, there is at present a conspicuous lack of reliable quantitative information on this issue.

To address the question of the relation between age, vaccination status, severity of an infection, and the likelihood of notification, we are currently investigating the immune status of the Dutch population at large by analyzing pertussis specific IgA and $\operatorname{IgG}$ antibody titers [29, 30], longitudinal trends in antibody titers in individual patients after a clinically recognized infection, and by clinically assessing the severity of $B$. pertussis infections in relation to age and vaccination status. Together the results of these analyses should give more insight in the relation between the age and immune status of an individual, the probability of infection, the probability of notification, and the severity of infection. 


\section{Vaccination against an evolving $B$. pertussis population}

There is increasing molecular evidence that in comparison with the pre-vaccination era $B$. pertussis strains circulating in the Netherlands have changed in a number of virulence factors [14, 15]. A comparison of the pertactin and pertussis toxin variants present in the currently circulating strains of B. pertussis with the variants present in the Dutch vaccine show that there is at present a mismatch between the two. It is likely that this mismatch has some adaptive significance. In fact, there are indications that the older, 'vaccine' strain is found relatively more often in unvaccinated individuals in comparison with the newly arising strains [14]. This implies that the protection induced by the vaccine appears to be less strong against the new strains than it was against the vaccine strain. Whether cross-immunity between the old and new $B$. pertussis strains is also incomplete is at present not known.

The fact that the $B$. pertussis population may have evolved to circumvent the immune response elicited by the vaccine raises a number of questions concerning the composition and use of future vaccines like: Which combination of antigens protects optimally against the circulation strains? How should one balance the breadth against the depth of a vaccine (i.e. should one include many polymorphic antigens or only a few monomorphic antigens)? Which vaccines, vaccination schemes and coverages minimize the probability of appearance of escape mutants? How does the evolutionary pressure on the $B$. pertussis population depend on the relationship between natural and vaccine-induced immunity? Should the aim of vaccination be to reduce symptomatic disease while maintaining circulation, or should it be to reduce the infectiousness of the pathogen population? Such evolutionary questions are just beginning to be addressed by theoreticians and empiricists alike. Careful confrontation of model predictions with reallife data is the logical next step. The ultimate goal is, of course, the application of the insights so obtained to the development and use of future vaccines.

\section{ACKNOWLEDGEMENTS}

We thank an anonymous referee for constructive criticism. Dr André de Roos is gratefully acknowledged for providing the simulation package in which the model was implemented.

\section{APPENDIX: MODEL EQUATIONS AND ANALYSIS}

The dynamics of the model is given by the following set of partial differential equations (PDEs) for the ageand time-dependent variables:

$$
\begin{aligned}
\frac{\partial S_{1}(a, t)}{\partial t}+\frac{\partial S_{1}(a, t)}{\partial a}= & -\nu(a) S_{1}(a, t)-\lambda(a, t) S_{1}(a, t) \\
& -\mu(a) S_{1}(a, t) \\
\frac{\partial V(a, t)}{\partial t}+\frac{\partial V(a, t)}{\partial a}= & \nu(a) S_{1}(a, t)-\sigma_{V} V(a, t) \\
& -\mu(a) V_{1}(a, t) \\
\frac{\partial I_{1}(a, t)}{\partial t}+\frac{\partial I_{1}(a, t)}{\partial a}= & \lambda(a, t) S_{1}(a, t)-\rho_{1} I_{1}(a, t) \\
& -\mu(a) I_{1}(a, t) \\
\frac{\partial S_{2}(a, t)}{\partial t}+\frac{\partial S_{2}(a, t)}{\partial a}= & \sigma_{V} V(a, t)+\sigma_{I} R(a, t) \\
& -\lambda(a, t) S_{2}(a, t)-\mu(a) S_{2}(a, t) \\
\frac{\partial I_{2}(a, t)}{\partial t}+\frac{\partial I_{2}(a, t)}{\partial a}= & \lambda(a, t) S_{2}(a, t)-\rho_{2} I_{2}(a, t) \\
& -\mu(a) I_{2}(a, t) \\
\frac{\partial R(a, t)}{\partial t}+\frac{\partial R(a, t)}{\partial a}= & \rho_{1} I_{1}(a, t)+\rho_{2} I_{2}(a, t) \\
& -\sigma_{I} R(a, t)-\mu(a) R(a, t)
\end{aligned}
$$

Total population size $N(a, t)$ is given by the sum over all variables. Hence,

$\frac{\partial N(a, t)}{\partial t}+\frac{\partial N(a, t)}{\partial a}=-\mu(a) N(a, t)$.

Since all individuals are born susceptible, and since we assume that total population size remains constant, this implies that the only non-trivial boundary condition should satisfy

$S_{1}(0, t)=\int_{0}^{L} \mu(a) N(a, t) d a$,

i.e. deaths are exactly balanced by births.

The age- and time-dependent force of infection $\lambda(a, t)$ is given by

$\lambda(a, t)=\int_{0}^{L} c\left(a, a^{\prime}\right)\left(\beta_{1} I_{1}\left(a^{\prime}, t\right)+\beta_{2} I_{2}\left(a^{\prime}, t\right) d a^{\prime}\right.$. 
Here the contact function $c\left(a, a^{\prime}\right)$ is a measure for the number of contacts that an infected individual of age $a^{\prime}$ makes with a susceptible individual of age $a$, while the parameters $\beta_{1}$ and $\beta_{2}$ are the probability of transmission in a contact of a susceptible individual with a primary and secondary infected individual, respectively.

To determine the force of infection, it is assumed that the population is in the endemic equilibrium in the 1988-95 period. As a consequence, the force of infection is time-independent in this period (i.e. $\lambda(a, t)$ $=\lambda(a)$ ), and can be estimated using the endemic equilibrium of the model (A1) and the incidence data of Figure 1 [16]. Subsequently, given some assumptions on the form of the contact function, the force of infection in the pre-epidemic period can be used to obtain numerical estimates for the contact function [18]. In the present study we made the particularly simple assumption that the contact function has the form $c\left(a, a^{\prime}\right)=f(a) f\left(a^{\prime}\right)$. This is commonly referred to as the 'proportionate mixing' assumption. There are several reasons why one should not blindly trust the numerical results obtained with the methods outlined above. A strong point of the estimation procedure of the force of infection is that estimates of the force of infection are at least consistent with the dynamical model (A1). We refer to van Boven et al. [16] for a description of the procedure to estimate the force of infection, and for a discussion of the pros and cons of the method. General methods to estimate agespecific forces of infection are presented in [31]. Hethcote [18] gives a clear account on the determination of the contact function from a given force of infection.

In principle, various methods are available to numerically integrate the PDEs (A1). However, in our case the procedures are somewhat more complex than usual, as the boundary condition $S_{1}(0, t)$ depends on the variables through total population size $N(a, t)$. To address this complication, a specific method has been developed, the so-called Escalator Boxcar Train. An intuitive derivation is given in [32], while a formal derivation is presented in [33].

\section{REFERENCES}

1. Bentsi-Enchill AD, Halperin SA, Scott J, et al. Estimates of the effectiveness of a whole-cell pertussis vaccine from an outbreak in an immunized population. Vaccine 1997; 15: 301-6.

2. Bass JW, Wittler RR. Return of epidemic pertussis in the United States. Pedriatic Infect Dis J 1994; 13: $343-5$.

3. Christie CDC, Marx ML, Marchant CD, et al. The 1993 epidemic of pertussis in Cincinatti. Resurgence of disease in a highly immunized population of children. New Engl J Med 1994; 331 : 16-21.

4. Deen JL, Mink CM, Cherry JD, et al. Household contact study of Bordetella pertussis infections. Clin Infect Dis 1995; 21: 1211-9.

5. Fine PEM, Clarkson JA. Reflections on the efficacy of pertussis vaccines. Rev Infect Dis 1987; 9: 866-83.

6. de Melker HE, Conyn-van Spaendonck MAE, Schellekens JFP. The pertussis epidemic in 1996; description and evaluation based on surveillance data from 1976 to 1996. Report no. 128507005, National Institute of Public Health and the Environment, Bilthoven, 1997.

7. de Melker HE, Conyn-van Spaendonck MAE, Rümke $\mathrm{HC}$, et al. Pertussis in the Netherlands: An outbreak despite high levels of immunization with whole-cell vaccine. Emerg Infect Dis 1997; 3: 175-8.

8. Mink CM, Cherry JD, Christenson PD, et al. A search for Bordetella pertussis infection in university students. Clin Infect Dis 1992; 14: 464-71.

9. Schmitt-Grohe S, Cherry JD, Heininger U, et al. Pertussis in German adults. Clin Infect Dis 1995; 21 : 860-6.

10. von Konig CHW, Postels-Multani S, Bock HL, et al. Pertussis in adults: frequency of transmission after household exposure. Lancet 1995; 346: 1326-9.

11. Gustaffson L, Hallander HO, Olin P, et al. A controlled trial of a two-component acellular, a five-component acellular, and a whole-cell pertussis vaccine. New Engl J Med 1996; 334: 349-55.

12. Simondon F, Preziosi MP, Yam A et al. A randomized double-blind trial comparing a two-component acellular to a whole-cell pertussis vaccine in Senegal. Vaccine 1997; 15: 1606-12.

13. Stehr K, Cherry JD, Heiniger U et al. A comparative efficacy trial in Erlangen, Germany in infants who received either the Lederle/Takeda acellular pertussis component DTP (DTaP) vaccine, the Lederle wholecell component DTP vaccine or DT vaccine. Pediatrics 1998; 101: 1-1.

14. Mooi FR, van Oirschot $\mathrm{H}$, Heuvelman $\mathrm{K}$, et al. Polymorphism in the Bordetella pertussis virulence factors P.69/pertactin and pertussis toxin in the Netherlands: temporal trends and evidence for vaccinedriven evolution. Infect Immun 1998; 66: 670-5.

15. van Loo IHM, van der Heide HGJ, Nagelkerke NJD, et al. Temporal trends in the population structure of Bordetella pertussis during 1949-1996 in a highly vaccinated population. J Infect Dis 1999; 179: 915-23.

16. van Boven M, de Melker HE, Schellekens JFP, et al. Waning immunity and sub-clinical infection in an epidemic model: implications for pertussis in the Netherlands. Math Biosciences 2000; 164: 161-82.

17. Anderson RM, May RM. Infectious diseases of humans. Oxford: Oxford University Press, 1991. 
18. Hethcote HW. Modeling heterogeneous mixing in infectious disease dynamics. In: Models for infectious human diseases: Their structure and relation to data. Isham V, Medley G, eds. Cambridge: Cambridge University Press, 1996: 215-38.

19. Grenfell BT, Anderson RM. Pertussis in England and Wales: an investigation of transmission dynamics and control by mass vaccination. Proc Royal Soc Lond B 1986; 236: 213-52.

20. Hethcote HW. An age-structured model for pertussis transmission. Math Biosciences 1997; 145: 89-136.

21. Hethcote HW. Simulations of pertussis epidemiology in the United States: effect of booster vaccinations. Math Biosciences 1999; 158: 47-73.

22. Long SS, Lischner HW, Deforest A, et al. Serologic evidence of sub-clinical pertussis in immunized children. Ped Infect Dis J 1990; 9: 700-5.

23. Long SS, Welkon CJ, Clark JL. Widespread silent transmission of pertussis in families: antibody correlates of infection and symptomatology. J Infect Dis 1990; 161: 480-6.

24. Sutter RW, Cochi SL. Pertussis hospitalizations and mortality in the United States, 1985-1988. Evaluation of the completeness of national reporting. JAMA 1992; 267: 386-91.

25. He QS, Viljanen MK, Nikkari S, et al. Outcomes of Bordetella pertussis infection in different age groups of an immunized population. J Infect Dis 1994; 170: 873-7.
26. Deville JG, Cherry JD, Christenson PD, et al. Frequency of unrecognized Bordetella pertussis infections in adults. Clin Infect Dis 1995; 21 : 639-42.

27. Farrington CP. The measurement and interpretation of age-specific vaccine efficacy. Int J Epidemiol 1992; 21 : 1014-20.

28. Ramsay MEB, Farrington CP, Miller E. Age-specific efficacy of pertussis vaccine during epidemic and nonepidemic periods. Epidemiol Infect $1993 ; 111$ : 41-8.

29. de Melker HE, Conyn-van Spaendonck MAE. Immunosurveillance and the evaluation of national immunization programmes: a population based approach. Epidemiol Infect 1998; 121: 637-43.

30. de Melker HE, Seroepidemiology of diphteria, tetanus, poliomyelitis, and pertussis. Evaluation of the National Immunisation Programme in the Netherlands. $\mathrm{PhD}$ thesis, Bilthoven, 1999.

31. Farrington CP, Kannan MN, Gay NJ. Estimation of the basic reproduction number for infectious diseases from age-stratified serological survey data. Appl Statist 2001; 50, in press.

32. de Roos AM. A gentle introduction to models of physiologically structured populations. In: Structurespopulation models in marine, terrestrial, and freshwater systems, Tuljapurkar S, Caswell H, eds. New York: Chapman \& Hall, 1997; 119-204.

33. de Roos AM. Numerical methods for structured population models: the Escalator Boxcar Train method. Num Meth Partial Diff Eq 1988; 4: 173-95. 